\title{
REALISMO E INDETERMINATEZZA NEI FONDAMENTI DELLA MATEMATICA
}

\author{
FRANCESCA BOCCUNI (*)
}

\begin{abstract}
SunTO. - Due dei maggiori indirizzi fondazionali in filosofia della matematica degli ultimi trent'anni, i.e. il neologicismo e lo strutturalismo matematico ante rem soffrono di problemi di indeterminatezza metafisica e semantica. Nel presente articolo, intendo fornire una disamina della causa di questo fenomeno e una conseguente soluzione in termini di riferimento arbitrario.
\end{abstract}

$$
* * *
$$

ABSTRACT. - Two of the most influential foundational approaches in the philosophy of mathematics of the past thirty years, i.e. neologicism and ante rem mathematical structuralism, suffer from metaphysical and semantic indeterminacy. My present aim is to offer an explanation of this phenomenon and provide a solution in terms of arbitrary reference.

\section{INTRODUZIONE}

La maggior parte dei contributi presenti in questo volume riguarda alcuni temi fondamentali per la fisica e la sua filosofia. Ad essi si allaccia in via efficace un tema maggiormente caro alla filosofia della matematica, i.e. l'indispensabilità della matematica per le teorie scientifiche. Alcuni autori coinvolti in quest'ultimo dibattito ${ }^{1}$ hanno sostenuto che l'indispensabilità della matematica per le scienze è un argomento a favore del realismo platonista, i.e. di quella posizione che sostiene l'esistenza delle entità matematiche in quanto fattori di verità degli enuncia-

(*) Università Vita-Salute San Raffaele, Milano, Italia.

E-mail: boccuni.francesca@unisr.it

1 Mi riferisco in particolare a Quine e Putnam. 
ti matematici necessari per la formulazione e la verità delle teorie scientifiche. $^{2}$

Questo tema fornisce un naturale spunto di riflessione relativo a una domanda pregressa che riguarda lo statuto della matematica stessa e che può essere efficacemente declinata in tre questioni fondamentali: l'esistenza delle entità matematiche (domanda di natura ontologica); la loro natura (domanda di natura metafisica); la nostra capacità di conoscere verità su di esse (domanda di natura epistemologica). Che queste tre questioni abbiano tradizionalmente accompagnato l'indagine sui fondamenti della matematica, si può facilmente intuire riflettendo su alcune considerazioni di quello che è considerato il padre fondatore, fra altre discipline filosofiche, della filosofia della matematica moderna: Gottlob Frege. Il contesto intellettuale in cui Frege inizia la sua ricerca sui fondamenti, nella seconda metà dell'Ottocento, è estremamente stimolante. All'inizio del XIX secolo, la geometria euclidea, che fino ad allora aveva rappresentato il paradigma di assoluta certezza matematica, perde il proprio status teorico privilegiato a causa dell'invenzione $\mathrm{o}$, se si preferisce, della scoperta - delle geometrie non euclidee. Durante i primissimi anni di docenza di Frege a Jena, a Halle Georg Cantor sviluppa il nucleo fondamentale della teoria degli insiemi. Si inaugura in quello stesso periodo la teoria dei numeri complessi. Tuttavia, a questo straordinario sviluppo, secondo Frege, non si accompagna l'altrettanto necessaria preoccupazione, di natura filosofica, di chiarire in modo inequivocabile la natura di quelle entità attorno a cui ruota l'indagine matematica. Frege lamenta la mancanza di una definizione esplicita delle nozioni matematiche non solo fondamentali ma, soprattutto, apparentemente più semplici, come ad esempio quelle di funzione, limite e numero naturale. Se l'indagine matematica stessa manca di fornire una definizione della natura delle nozioni cardine su cui si fonda, ecco che l'edificio matematico posa su basi incerte. Primo compito, dunque, secondo Frege, è quello di fondare la matematica su basi sicure, a cominciare dall'aritmetica, in quanto scienza matematica fra le più basilari, fornendo definizioni esplicite delle nozioni aritmetiche più elementari e rispondendo così alle fondamentali domande teo-

2 L'argomento di indispensabilità, in realtà, è stato proposto in versioni molto differenti. Quella che ho brevemente richiamato è quella più forte. Cfr. [8] in questo volume, ma anche [20]. 
riche che cosa sono $i$ numeri e come ci vengono dati? Non $^{3}$, dunque, una scelta peregrina quella di dedicare questo articolo alle tre menzionate questioni relative a esistenza e natura dei numeri naturali e conoscibilità della verità degli enunciati che vertono su di essi, in particolare rispetto ai due indirizzi fondazionali più influenti degli ultimi trent'anni in filosofia della matematica: il neologicismo ${ }^{4}$ e lo strutturalismo matematico (ante rem).

Tanto il neologicismo quanto lo strutturalismo ante rem sono indirizzi di orientamento realista platonista rispetto all'esistenza delle entità matematiche. Entrambi sostengono inoltre l'idea che le entità matematiche possano vedere la propria natura svelata da opportuni sistemi assiomatici e che, dunque, la loro conoscibilità sia da tali assiomi garantita. Le inevitabili distinzioni fra questi due orientamenti fondazionali saranno oggetto della §2. Vedremo inoltre, nella §3, che entrambi questi indirizzi, con buona pace dei loro sostenitori e di Frege, sono minati da problemi di indeterminatezza semantica e metafisica.

\section{Neologicismo e Strutturalismo matematico ante REM}

Fra i principi che guidano l'indagine neologicista, e per gli scopi del presente articolo, è utile ricordare i seguenti: l'idea neologicista che l'aritmetica debba essere ridotta alla mera logica o, quanto meno, a principi di natura analitica; ${ }^{6}$ l'idea che i numeri naturali esistano e siano oggetti matematici sui generis, dotati di una natura peculiare epistemicamente svelata dai principi che li governano. In questa sezione, dedicherò più spazio alla proposta assiomatica neologicista, in quanto presumibilmente meno intuitiva per un pubblico di non addetti ai lavori.

Il principio analitico su cui si fonda l'intero programma neologicista è il cosiddetto Principio di Hume [HP]. HP appartiene a quella

\footnotetext{
3 Cfr. [10].

4 In primis, [12] e [29].

5 In particolare, [23].

6 Questo assunto è in continuità con il programma fondazionale fregeano. Benché il nucleo fondamentale del presente articolo abbia importanti ricadute anche su questo aspetto del programma neologicista, rimando alla sezione $\$ 5$ per delle considerazioni generali a questo proposito e a [4] per considerazioni di dettaglio.
} 
classe di principi noti come principi di astrazione che, in filosofia della matematica, tradizionalmente vengono fatti risalire a Frege. Un principio di astrazione è un principio della forma:

$$
\S e_{1}=\S e_{2} \leftrightarrow E\left(e_{1}, e_{2}\right)
$$

dove $\S$ è un operatore che mappa entità di tipo $e_{1}, e_{2}$ in entità di un tipo (potenzialmente) diverso ed $E$ è una relazione di equivalenza che vige fra $e_{1}, e_{2}$. Intuitivamente, un principio di astrazione dice che l'astratto di $e_{1}$ è identico all'astratto di $e_{2}$ sse $e_{1}$ e $e_{2}$ stanno nella relazione di equivalenza $E$.

Esempi noti di tali principi sono i seguenti:

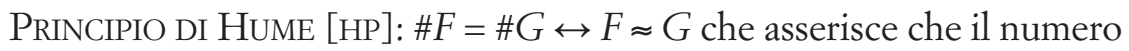
degli oggetti che cadono sotto il concetto $F$ è identico al numero degli oggetti che cadono sotto il concetto $G$ sse esiste una corrispondenza biunivoca fra $F$ e $G .^{7}$

LEGGE V [BLV]: $\{x: F x\}=\{x: G x\} \leftrightarrow \forall x(F x \leftrightarrow G x)$ che asserisce che l'estensione del concetto $F$ è identica all'estensione del concetto $G$ sse $F$ e $G$ sono coestensivi; ${ }^{8}$

PRINCIPIO DELLE DIREZIONI: $d(a)=d(b) \leftrightarrow a / / b$ che asserisce che le direzioni delle rette $a$ e $b$ sono identiche sse $a$ è parallela a $b{ }^{9}$

Come detto, HP è il principio fondamentale del programma neologicista. Dal punto di vista matematico, HP interpreta l'aritmetica di Peano al secondo ordine; ${ }^{10}$ esso inoltre, a differenza di BLV, è consistente. ${ }^{11}$

$7 \quad[10], \S 62$.

$8 \quad[11]$, vol. I §20.

9 [10], §65. Gli operatori introdotti da HP e BLV sono funzioni iniettive che vanno dall'insieme potenza $\wp(D)$ del dominio del primo ordine $D$ a $D$ stesso.

10 Assiomaticamente, il neologicismo si avvale della logica dei predicati del secondo ordine impredicativa e di HP. Ė possibile dimostrare che questa assiomatizzazione interpreta gli assiomi dell'aritmetica di Peano al secondo ordine PA². Cfr. e.g. [30].

11 Da BLV è possibile derivare il cosiddetto paradosso di Russell, i.e. il paradosso che concerne l'insieme che contiene tutti e soli gli insiemi che non appartengono a se stessi. 
Inoltre, HP è, per i neologicisti, una verità analitica della nozione di Numero Naturale. Infine, HP è, secondo i neologicisti, quel principio che non solo garantisce l'esistenza degli oggetti cui i termini singolari a sinistra del bicondizionale si riferiscono, ma ne svela la natura peculiare ultima permettendoci di individuare che tipo di oggetti i numeri naturali siano e che oggetto sia ciascun numero naturale e, inoltre, fornendoci, con ciò stesso, accesso epistemico ad essi.

Per lo strutturalista matematico ante rem, ${ }^{12}$ d'altro canto, l'aritmetica dei numeri naturali riguarda una struttura matematica astratta, i.e. la struttura dei numeri naturali, in cui ciascun numero naturale altro non è che una posizione nella struttura, esaurito nella sua natura ultima dalle proprietà e relazioni aritmetiche che vigono con le altre posizioni nella struttura. Dato che esiste un'unica struttura dei numeri naturali, ${ }^{13}$ esiste un'unica ennesima posizione saliente e, dunque, esiste un unico numero $n$.

Entrambi gli orientamenti, pur nelle loro radicali differenze,

1. assumono l'esistenza di entità matematiche - i numeri naturali, per il neologicista; la struttura dei naturali e le posizioni interne alla struttura, per lo strutturalista ante rem;

2. assumono di vederne svelata la natura ultima dai loro rispettivi assiomi - per il neologicista, i numeri naturali sono oggetti matematici sui generis, dotati di una natura peculiare svelata da HP; per lo strutturalista ante rem, le proprietà e relazioni aritmetiche strutturali costituiscono le posizioni nella struttura dei naturali, esaurendone così la natura.

Per entrambi gli indirizzi, la conoscibilità dei numeri naturali è basata sulla conoscenza degli assiomi che li governano.

12 A onor del vero, due sono i principali indirizzi strutturalisti, in reciproca opposizione: quello ante rem di Stewart Shapiro in e.g. [23]; quello eliminativista o in re di Hellman. Ai fini del presente articolo, sarà sufficiente prendere in considerazione il primo. Per ulteriori dettagli, cfr. [20].

13 Lo strutturalista ante rem infatti si avvale della logica dei predicati del secondo ordine impredicativa come logica di sfondo, per fornire un'assiomatizzazione della struttura dei numeri naturali, garantendo così la categoricità della sua teoria. Cfr. [23]. 


\section{INDETERMINATEZZA}

Notoriamente, tuttavia, HP non è in grado di fornire accesso epistemico alla natura intrinseca dei numeri naturali: benché da HP sia possibile derivare tutte le verità della forma $\# F=\# G{ }^{14}$ esso rimane silente relativamente alla verità $\mathrm{o}$ falsità di enunciati di identità misti della forma $\# F=q$, in cui $q$ non sia già dato come un termine singolare della forma \#blablabla, governato da HP. Questo è il cosiddetto problema di Giulio Cesare, già messo in evidenza da [10]: il valore di verità di un enunciato di identità della forma il numero delle sedie in questa stanza $=$ Giulio Cesare rimane indeterminato. ${ }^{15}$ In conseguenza, in base al solo HP, non siamo in grado di determinare il valore di verità di tutti gli enunciati di identità che contengono termini singolari numerici della forma $\# F$, in particolare non siamo in grado di determinare se $\# F=$ Giulio Cesare ${ }^{16}$ Per ciò stesso, se anche i numeri naturali fossero oggetti sui generis, così come vuole il neologicista, il principio che li governa non potrebbe svelarne pienamente la natura.

Questa forma di indeterminatezza può essere estesa anche a indirizzi fondazionali altri dal neologicismo, fino a mettere in discussione l'assunto secondo cui i numeri naturali siano oggetti con una natura peculiare. Se ad esempio si ritenesse che a fondamento dell'intero edificio matematico debba trovarsi la teoria degli insiemi, le verità relative agli oggetti aritmetici sarebbero ridotte a verità relative a insiemi. Tuttavia, si consideri il cosiddetto problema di Benacerraf: ${ }^{17}$ assumendo che una teoria insiemistica possa fornire una definizione dei numeri naturali, abbiamo a disposizione definizioni diverse. Secondo la defini-

14 E, cosa più importante, quelle della forma - (\#F=\#G).

15 Il problema di Cesare ha dato origine a una amplissima letteratura, sia di stampo fregeano sia di stampo neologicista. Cfr. e.g. [9], [10], [12], [13], [14], [17], [22], [26], [27], [29].

16 Auspicabilmente, il neologicista vuole rispondere negativamente al quesito posto dal problema di Cesare, in particolare vuole che siano falsi enunciati della forma '\#F = Giulio Cesare'. Tuttavia, se il problema di Cesare fosse risolto nel modo auspicato dal neologicista, conseguentemente HP sarebbe in grado di dimostrare verità relative a oggetti concreti, e.g. che un condottiero romano non è il successore di 1 , mettendo così in pericolo il desideratum neologicista in base al quale HP sarebbe un principio analitico del concetto di Numero Naturale.

17 Cfr. [2]. 
zione di Zermelo, ad esempio, il numero naturale 2 è definibile come $\{\{\varnothing\}\}$, ma secondo, la definizione di Von Neumann il numero naturale 2 è $\{\varnothing,\{\varnothing\}\},{ }^{18}$ causando così una forma di indeterminatezza relativa alla natura ultima dei numeri naturali.

Una risposta a prima vista definitiva al problema dell'indeterminatezza viene fornita dallo strutturalista ante rem. Come dicevo, in questa prospettiva, l'aritmetica riguarda la struttura astratta dei numeri naturali, in cui ciascun numero altro non è che una posizione nella struttura, esaurito nella sua natura ultima dalle proprietà e relazioni aritmetiche che vigono con le altre posizioni. Dato che esiste un'unica struttura dei numeri naturali, esiste un'unica posizione saliente che faccia da successore del successore di zero e, dunque, esiste un unico numero 2 .

In questo senso, il dubbio benacerrafiano viene meno. Tanto $\{\{\varnothing\}\}$ quanto $\{\varnothing,\{\varnothing\}\}$ sono possibili candidati ad occupare la posizione nella struttura dei numeri naturali che pertiene al numero 2. Essi infatti vengono definiti in assiomatizzazioni insiemistiche per l'aritmetica di Peano, ciascuna delle quali altro non è che una possibile istanza della struttura astratta dei naturali. Tutto ciò che interessa è che proprietà e relazioni aritmetiche valgano in ciascun modello per l'aritmetica, che sia un modello zermeliano o vonneumanniano, e che dunque ciascuno di questi modelli verifichi gli assiomi dell'aritmetica, come del resto già accade. In conseguenza, lo strutturalista ante rem può proporre al neologicista una soluzione piuttosto semplice al problema di Cesare: se Giulio Cesare è un individuo di un dominio di quantificazione infinito numerabile che possa essere ordinato in una $\omega$-sequenza, allora Giulio Cesare può occupare la posizione che pertiene al numero 2 nella struttura dei numeri naturali, senza che con ciò siamo costretti a identificare metafisicamente un'entità matematica con un console della Res Publica romana.

Tuttavia, in base all'idea strutturalista secondo cui proprietà e relazioni strutturali dovrebbero costituire le posizioni in una struttura matematica, non è possibile distinguere fra certe posizioni in strutture che ammettano automorfismi non triviali, come ad esempio la struttura

18 Naturalmente, le teorie sottese alle due definizioni in questione forniscono modelli per l'aritmetica di Peano espressa in una logica dei predicati del primo ordine. In sostanza, queste due teorie sono vere degli stessi oggetti, anche se esse non sono in grado di stabilire in senso assoluto come questi oggetti siano fatti. 
dei numeri complessi. ${ }^{19}$ In [24] e [25], Shapiro cerca di risolvere questa forma di indeterminatezza, assumendo che i termini che si riferiscono alle posizioni indiscernibili, e.g. ' $\checkmark-1$ ': $i$ e $-i$, debbano essere trattati come parametri o nomi arbitrari. Ai termini per indiscernibili viene assegnato un denotato arbitrario e, dunque, ogniqualvolta fosse necessario fornire un denotato a ' $\sqrt{-}-1$ ', questo denotato verrà scelto arbitrariamente fra $i$ e $-i$, a seconda del contesto. Questa soluzione, però, impone un doppio standard al trattamento semantico dei termini singolari, utilizzato per risolvere esclusivamente il problema degli indiscernibili: il numerale ' 2 ' si riferisce in modo univoco ad una certa posizione nella struttura dei naturali, mentre ' $\sqrt{ }-1$ ' si riferisce arbitrariamente a uno dei suoi due valori, $i$ oppure $-i$.

Se dunque, da un lato, l'approccio neologicista soffre di una forma di indeterminatezza semantica e metafisica relativa al suo assioma non logico fondamentale HP, l'approccio strutturalista ante rem, che a prima vista sembra una soluzione intuitiva a problemi quale il problema di Cesare, non è da meno, nel momento in cui si considerassero casi più complessi della semplice aritmetica.

Dal mio punto di vista, il problema dell'indeterminatezza, tanto per il neologicista quanto per lo strutturalista ante rem, nasce da una certa lettura dei termini singolari, quali ' $\# F$ ' o il numerale ' 2 ', sottesa a questi indirizzi fondazionali. Tale lettura si basa sull'assunto che le costanti individuali matematiche e, in generale, $i$ termini singolari matematici, funzionino (o debbano funzionare) in modo analogo ai nomi propri nel linguaggio naturale, nella misura in cui, affinché un termine singolare matematico sia referenziale, e dunque la verità delle asserzioni matematiche abbia una semantica at face value, ci viene richiesto di individuare (single out), all'interno di un dato dominio, un individuo che ci faccia da referente di quel dato termine. Chiamerò questa forma di riferimento attraverso individuazione riferimento canonico. ${ }^{20}$

Basandomi sul lavoro congiunto [4], intendo

1. rigettare l'assunto, comune al neologicista e allo strutturalista ante rem, sulla semantica dei termini singolari in matematica;

19 Il dibattito sul fenomeno degli indiscernibili e il suo impatto sullo strutturalismo matematico ante rem è molto ampio. Per alcuni riferimenti, cfr. [7], [15], [23] .

20 Per la nozione di 'individuazione', cfr. [16]. 
2. applicare una proposta di soluzione al caso del neologicismo, risolvendo così il problema di Cesare.

Intendo sostenere che, benché ' $\# F$ ' sia genuinamente referenziale, la forma di riferimento incorporata in ' $\# F$ ' può essere interpretata come una forma di riferimento arbitrario. ${ }^{21}$ Grazie all'uso del riferimento arbitrario, è possibile evitare i problemi di indeterminatezza connessi con il neologicismo e causati, a mio avviso, dal riferimento canonico e, tuttavia, continuare a utilizzare HP in una semantica classica. In conseguenza, anche lo strutturalista ante rem potrà avvalersi della presente soluzione, fornendo così una semantica univoca e uniforme per i termini singolari, senza dover proporre una soluzione ad hoc al problema degli indiscernibili e della indeterminatezza semantica. ${ }^{22}$

\section{RIFERIMENTO ARBITRARIO}

In alcuni articoli relativamente recenti, ${ }^{23}$ è stato sostenuto che (alcuni) termini singolari nel discorso matematico vadano intesi come parametri o nomi arbitrari: come termini che funzionano grammaticalmente come termini singolari, ma che semanticamente funzionano come pronomi o descrizioni indefinite. Ad esempio, in ambito matematico, termini di questo genere possono essere introdotti da locuzioni della forma Sia n un arbitrario numero naturale.

Questa posizione ha il deciso vantaggio di essere stata proposta su basi semantiche o linguistiche (dunque, non metafisiche), ma può essere declinata in modi molto differenti, anche fra loro incompatibili. [5] fornisce una carrellata delle possibili opzioni in gioco, secondo cui è possibile sviluppare due distinte spiegazioni del riferimento in ambito matematico.

Secondo una prima visione, si può sostenere una posizione liberale e ritenere che esistano due nozioni indipendenti di riferimento:

21 Sulla falsariga di [24] e [25].

22 Quest'ultimo aspetto non sarà analizzato dettagliatamente, per motivi di spazio.

23 Cfr. e.g. [5], [21], [23], [24], [28]. 
(a) il riferimento canonico - riferimento tramite termini singolari come i nomi propri operato attraverso individuazione;

(b) il riferimento arbitrario come forma di riferimento altra rispetto a quello canonico.

In base alla seconda opzione presentata da [5], è possibile invece assumere una posizione critica sul problema del riferimento e sostenere, alternativamente, che:

(i) (almeno alcuni) termini singolari sono parametri e non sono referenziali - e dunque l'unica forma di riferimento genuino per i termini singolari è il riferimento canonico; ${ }^{24}$

(ii) tutti i termini singolari hanno riferimento genuino, tuttavia, non (necessariamente) tramite individuazione: ciò che è realmente basilare è il riferimento arbitrario - e, se proprio lo si vuole, il riferimento canonico può essere costruito a partire da esso.

La mia opzione prediletta è la (ii): relativamente ai termini della forma ' $\# F$ ', si dà riferimento genuino, ma si può evitare l'ingerenza della nozione di individuazione. Grazie a questa prospettiva, il riferimento arbitrario viene considerato genuino, per esso viene fornita una semantica che risolve il problema di Cesare per il neologicista, la semantica at face value delle asserzioni matematiche viene preservata e non è necessario, a differenza di quanto propone lo strutturalista ante rem, istituire un doppio standard di trattamento semantico dei termini singolari per gestire l'indeterminatezza. In particolare, il riferimento senza individuazione rende possibile sostenere che non c'è necessità di individuare i valori semantici dei termini della forma ' $\# F$ ', per avere una nozione di riferimento che renda conto del loro funzionamento semantico. Nel dominio, possiamo avere quel che ci pare: numeri, sedie, una serie infinita di repliche di Giulio Cesare, tavoli o boccali di birra, per dirla á la Hilbert, posto che ce ne siano in quantità sufficiente per fornire referenti ai termini singolari della forma ' $\# F$ '. La verità di $\mathrm{HP}$, dunque, sarà solo questione di cardinalità del dominio ${ }^{25}$ e non del

24 Questa posizione è sostenuta da [21], [23], [24].

25 HP richiede che il dominio sia infinito numerabile. 
tipo di individui che lo abitano: la nostra semantica sarà totalmente indifferente alla loro natura ultima.

\subsection{Arbitrarietà}

A questo punto, tuttavia, è necessario spiegare in cosa consista l'arbitrarietà. Alcuni autori ${ }^{26}$ considerano il riferimento arbitrario come primitivo e genuinamente referenziale, mentre l'arbitrarietà in esso coinvolta come una caratteristica di natura epistemica.

Logici e matematici spesso usano locuzioni della forma Sia $n$ un numero naturale arbitrario. Questo genere di locuzioni introducono il riferimento a, e.g., un arbitrario numero naturale $n$ e, secondo [6], sono governate dal seguente principio del Riferimento Arbitrario:

RA: È possibile fissare il riferimento di un'espressione arbitrariamente. Quando ciò avviene, l'espressione riceve il suo valore semantico usuale, sebbene non sappiamo e non possiamo sapere che valore in particolare riceva. ${ }^{27}$

Secondo [18], questa possibilità di riferirsi, almeno in via non canonica, a un'entità di un dominio di quantificazione è presupposta dal ragionamento logico-matematico di stampo classico, anche quando si considerino domini non numerabili.

Questo tipo di riferimento è catturato dal ruolo cruciale che il riferimento arbitrario gioca nel ragionamento formale e informale. Il riferimento arbitrario, infatti, esibisce due caratteristiche logiche distinte, che lo rendono essenziale per le derivazioni: l'arbitrarietà e la determinatezza. Tramite il riferimento arbitrario, possiamo considerare una qualsiasi entità di un dominio: dato che il riferimento arbitrario non richiede individuazione, il nome arbitrario ' $a$ ' non si riferisce a una entità $a$ individuata, ma si riferisce a una arbitraria entità $a$ di quel dominio. Conseguentemente, l'ignoranza epistemica relativa a quale entità $a$ sia conserva la validità generale delle inferenze su $a$. Allo stesso tempo, tuttavia, nelle inferenze su $a$, ' $a$ ' deve denotare la medesima entità, distinta da tutte le altre entità del dominio, per preservare la vali-

\footnotetext{
26 [3], [6], [18], [19].

27 [6]. Tr. it. mia.
} 
dità stessa delle inferenze. In questo senso, un'espressione arbitraria assume un'entità particolare come suo valore semantico, tuttavia non sappiamo e non possiamo sapere quale valore essa assuma.

Questa posizione ha il vantaggio di fornire motivazione per le usuali restrizioni alle regole di introduzione ed eliminazione dei quantificatori in deduzione naturale. E.g., nella applicazione della regola di Introduzione dell'Universale [IU], quando si passa da $\phi(a)$ a $\forall x \phi x$, viene richiesto che nessuna delle assunzioni da cui l'universale introdotto dipende contenga il nome arbitrario $a$. Questo perché, per concludere che tutti gli $x$ sono $\phi$ da $\phi(a)$, dobbiamo essere certi che nessuna assunzione concernente specifiche proprietà di $a$ sia presente. Sembra quindi che una forma di ignoranza epistemica sia qui in gioco e che sia questa ignoranza epistemica relativamente alle proprietà precipue di $a$ a rendere possibile il corretto uso di IU. ${ }^{28}$

\subsection{Semantica}

Come rendere formalmente, tuttavia, quest'intuizione concernente il riferimento arbitrario, in particolare per i termini singolari della forma ' $\# F$ ' governati da HP?

La semantica proposta è fornita nei termini della nozione di invarianza sotto isomorfismi. Sul mercato, esistono due nozioni di invarianza. ${ }^{29}$ Una prima nozione di invarianza guarda all'entità particolare dell'appropriato tipo semantico individuato da una espressione relativamente a un dominio. Tuttavia, in molti casi, un dominio non restituisce un singolo oggetto come denotato di un'espressione, ma un range di oggetti candidati del tipo semantico in questione - e.g. $i$ e $-i$. L'intero range esaurisce la funzione semantica dell'espressione: per quanto ne sappiamo, uno qualsiasi dei candidati particolari può farci da referente ed è in base a questa intuizione che è possibile fornire una semantica per il riferimento arbitrario. In casi di questo tipo, infatti, sembra ugualmente saliente considerare solo l'invarianza dell'insieme dei candidati referenti, in base alla nozione che [28] chiama invarianza debole. Quando questo insieme di candidati è invariante, la funzione semantica di un'espressione è immune alla natura degli oggetti nel dominio.

28 Analogamente per la regola di Elimazione Esistenziale.

29 [28]. 
Nella visione epistemica del riferimento arbitrario, un nome arbitrario denota un particolare elemento dell'insieme dei candidati del tipo semantico appropriato. Ma dato che non è possibile sapere quale elemento in particolare, l'intero insieme di candidati servirà a esaurire la funzione semantica del nome arbitrario in questione. Possiamo quindi definire la nozione di invarianza debole per il riferimento arbitrario epistemico come segue:

Un'espressione $\phi$ [e.g. \#] è debolmente invariante sse, per ogni dominio $D$, $D^{\prime}$ e biiezione $i$ da $D$ a $D^{\prime}$, l'insieme delle denotazioni candidate $\phi *=$ $\{\gamma: \gamma$ è una denotazione candidata per $\phi$ su $D\}$ è tale che $i^{+}(\phi *)=\{\gamma: \gamma$ è una denotazione candidata per $\phi$ su $\left.D^{\prime}\right\}$.

Intuitivamente, questo dice che un'espressione ' $\phi$ ' è debolmente invariante nel caso in cui, iniziando con l'insieme delle denotazioni candidate per ' $\phi$ ' su un dominio $D$ e rimpiazzando sistematicamente gli oggetti in $D$ con gli oggetti in $D$ ', si ottiene quello che avremmo ottenuto semplicemente prendendo in considerazione l'insieme delle denotazioni candidate per ' $\phi$ ' su $D$. Sarebbe quindi naturale applicare la medesima considerazione agli oggetti in un dominio del primo ordine $D$ che la funzione \# prende come valori. Tuttavia, in questo modo, \# si comporterebbe come una funzione di scelta: per ogni dominio $D$, il denotato di ' $\# F$ ' in $D$ è un arbitrario individuo scelto a caso da \# in un sottoinsieme qualsiasi di $D$. Purtroppo, nessun operatore di astrazione che abbia come dominio $\wp(D)$ e codominio $D$, se $D$ contiene almeno due oggetti, è una funzione di scelta, pena la contraddizione. ${ }^{30}$ La soluzione prescelta sta dunque nel considerare in base al riferimento arbitrario e all'invarianza debole non i candidati referenti per ' $\# F$ ', bensì innanzitutto i candidati referenti per '\#'. Il riferimento della funzione di astrazione, dunque, che dovrebbe catturare la nozione di Numero Naturale è interpretata arbitrariamente - arbitrarietà viene poi ereditata dai termini singolari costituiti tramite applicazioni di \#. In questo senso, la denotazione di \# su un dominio $D$ è una qualsiasi funzione da $\wp(D)$ a $D$ che soddisfi HP.

In che modo la visione epistemica del riferimento arbitrario permette di risolvere il problema di Cesare per il neologicismo? 
Assumendo che Cesare sia un elemento di un dominio $D$, Cesare può essere il referente di ' $\# F$ '. $\mathrm{Ma}$, per riferimento arbitrario, nessuna della proprietà precipue di Cesare, essenziali o meno che siano, gioca un ruolo nel fissare il riferimento di ' $\# F$ ', dunque, non ha neppure senso chiedersi se Cesare realmente sia o meno, e.g. il numero degli $F$. Ciò che conta è che, se Cesare è un elemento di un dominio $D$ su cui HP è interpretato, Cesare può essere scelto come denotato del termine numerico ' $\# F$ ', per giocare il ruolo del numero degli $F$. Del resto, nessun oggetto particolare gioca un ruolo nello specificare la denotazione di ' $\# F$ '. Per l'approccio epistemico, tutto ciò che possiamo sapere in base a HP riguarda le proprietà e relazioni aritmetiche comuni a qualsiasi scelta di referente arbitrario per \#. In questo senso, questa posizione preserva un ingrediente chiave della posizione strutturalista ante rem, secondo cui le proprietà aritmetiche valgono indipendentemente dalla natura degli oggetti che giocano il ruolo strutturale appropriato e i fatti aritmetici (conosciuti) sono tipicamente insensibili alla natura peculiare degli oggetti in un dato dominio, che siano condottieri romani, tavoli o boccali di birra. A differenza, tuttavia, dello strutturalismo ante rem, la visione epistemica del riferimento arbitrario permette di evitare un doppio standard nel trattamento del riferimento dei termini singolari: non c'è bisogno di fornire a ' $\sqrt{ }-1$ ' un trattamento semantico diverso da quello riservato al numerale ' 2 '.

\subsection{Neologicismo strutturalista}

Come accennato nella §2, uno degli assunti fondamentali del programma neologicista è che l'aritmetica debba essere ridotta alla mera logica o, quanto meno, a principi di natura analitica. Nella fattispecie, HP dovrebbe essere un principio analitico della nozione di Numero Naturale. Tuttavia, l'indeterminatezza metafisica e semantica dovuta al problema di Cesare mette a repentaglio questa prospettiva: HP non può essere un principio analitico della nozione di Numero Naturale, sia che il problema di Cesare rimanga irrisolto e dunque HP non sia in grado di fissare il significato dei termini della forma ' $\# F$ ', sia che il problema di Cesare sia risolto come auspica il neologicista e quindi risultino falsi enunciati della forma $\# F=$ Giulio Cesare, dato che in quest'ultimo caso HP esprimerebbe delle verità relative a oggetti contingenti, la cui esistenza e natura sono pienamente indipendenti da HP - cosa che non è certamente un desideratum di un principio analitico. 
Il fatto di interpretare HP tramite una semantica per il riferimento arbitrario che faccia uso della nozione di invarianza debole permette di affermare che HP è un principio di natura logica. Infatti, come altri autori sostengono (e.g. Tarski, Sher, McGee, Sagi, Woods, Paseau), l'invarianza sotto isomorfismi viene intesa come condizione necessaria, benché non sufficiente, di logicità, in forza del fatto che una nozione logica è indifferente alla natura precipua degli oggetti nei diversi domini cui quella nozione si applica. L'invarianza debole cattura il modo in cui le espressioni che si riferiscono arbitrariamente sono immuni alla natura degli oggetti di un dominio. Se dunque anche l'invarianza debole, come credo, è condizione necessaria di logicità, gli operatori di astrazione come \# possono essere intesi come operatori logici, nella misura in cui si garantisca che la denotazione di \# su un dominio $D$ sia una qualsiasi funzione da $\wp(D)$ a $D$ che soddisfi HP.

Grazie all'uso della nozione di riferimento arbitrario e alla conseguente semantica per HP proposta sulla base della nozione di invarianza debole, è possibile proporre un indirizzo fondazionale alternativo sia al neologicismo sia allo strutturalismo ante rem, che mantenga alcuni assunti fondamentali di entrambi questi approcci e tuttavia ne eviti i difetti più evidenti - l'indeterminatezza semantica e il doppio standard nel trattamento semantico dei termini singolari. Questo nuovo indirizzo fondazionale, ampiamente presentato e difeso in [4], passa sotto il nome di NEOLOGICISMO STRUTTURALISTA, proprio per il fatto che, in base all'utilizzo della nozione di invarianza debole, HP si vede assegnato il ruolo di principio di natura logica (neologicismo) e, al tempo stesso, tutto ciò che è rilevante per determinare il riferimento dei termini singolari della forma ' $\# F$ ' sono le proprietà e relazioni aritmetiche (strutturalismo ante rem), dato che la natura precipua degli individui di un appropriato dominio di quantificazione è totalmente superflua per il trattamento semantico dei termini singolari e per la verità dei principi che li governano.

\section{ConClusioni}

Il neologicismo e lo strutturalismo matematico ante rem condividono una posizione realista platonista sull'esistenza delle entità matematiche e, tuttavia, assumono che tali entità abbiano natura peculiare, assunzione che causa fenomeni di indeterminatezza, così come implica- 
to, a mio parere, dal trattamento semantico che questi due approcci riservano ai termini singolari.

Lo scopo di questo articolo è stato salvare alcune ispirazioni di fondo di questi due indirizzi fondazionali, evitandone i problemi di indeterminatezza: in particolare, la genuina referenzialità dei termini singolari quali i numerali; l'idea che solo le proprietà e relazioni aritmetiche debbano essere soddisfatte da una qualsiasi struttura per i numeri naturali.

A questo scopo, ho proposto che espressioni quali 'il numero dei' non si riferiscano canonicamente, ma che comunque i termini singolari della forma ' $\# F$ ' siano termini singolari genuinamente, benché arbitrariamente, referenziali. In forza della semantica fornita per catturare questa intuizione, basata sulla nozione di invarianza debole, è stato infine possibile affermare che principi quali HP sono principi di natura logica, recuperando così uno dei desiderata fondamentali del programma neologicista. ${ }^{31}$

\section{BIBLIOGRAFIA}

[1] Antonelli Aldo, Notions of Invariance for Abstraction Principles, Philosophia Mathematica, 2010: 18: 276-292.

[2] Benacerraf Paul, What Numbers Could Not Be, Philosophical Review, 1965: 74: 47-73.

[3] Boccuni Francesca, Plural Logicism, Erkenntnis, 2013: 78: 1051-1067.

[4] Boccuni Francesca, Woods Jack, Structuralist Neologicism, Philosophia Mathematica, 2018: nky017, https://doi.org/10.1093/philmat/nky017.

[5] Brandom Robert, The Significance of Complex Numbers for Frege's Philosophy of Mathematics, Proceedings of the Aristotelian Society, 1996: 96: 293-315.

[6] Breckenridge Wylie, Magidor Ofra, Arbitrary reference, Philosophical Studies, 2012: 158: 377-400.

[7] Burgess John, Book Review: Stewart Shapiro. Philosophy of Mathematics: Structure and Ontology, Notre Dame Journal of Formal Logic, 1999: 40: 283-291.

[8] Cinti Enrico, Scienza e matematica: il caso dell'indispensabilità, Lost in physics and metaphysics. Questioni di realismo scientifico, Incontro di studio, 2019: 97: 69-79.

[9] Dummett Michael, Frege. Philosophy of Mathematics, Harvard University Press, Harvard, 1991.

[10] Frege Gottlob, Die Grundlagen der Arithmetik, Hildesheim: Georg Olms Verlagbuchhandlung, 1884; traduzione inglese a cura di J.L. Austin, The Foundations of Arithmetic, Blackwell, 1950.

$31 \mathrm{E}$, in fin dei conti, anche fregeano. 
[11] Frege Gottlob, Grundgesetze der Arithmetik, Jena: Verlag Hermann Pohle, Band I/II, 1893-1903. Traduzione inglese completa a cura di P. Ebert e M. Rossberg (con C. Wright), Basic Laws of Arithmetic: Derived using concept-script, Oxford University Press, Oxford, 2013.

[12] Hale Bob, Wright Crispin, The Reason's Proper Study: Essays Toward a NeoFregean Philosophy of Mathematics, Oxford University Press, New York, 2001.

[13] Heck Richard Jr., The Julius Caesar Objection, in: Language, Thought, and Logic: Essays in Honour of Michael Dummett, R. Heck Jr. (a cura di), 273-308, Oxford University Press, Oxford, New York, 1997.

[14] Heck Richard Jr., Julius Caesar and Basic Law V, Dialectica, 2005: 59: 161-178.

[15] Keränen Jukka, The Identity Problem for Realist Structuralism, Philosophia Mathematica, 2001: 9: 308-330.

[16] Lowe Edward Jonathan, Individuation, in: The Oxford Handbook of Metaphysics, M. J. Loux, D. W. Zimmerman (a cura di), 75-95, Oxford University Press, Oxford, New York, 2003.

[17] MacBride Fraser, The Julius Caesar Problem: More Problematic than Ever, in: Identity and Modality, F. MacBride (a cura di), 174-202, Clarendon Press, Oxford, 2006.

[18] Martino Enrico, Arbitrary Reference in Mathematical Reasoning, Topoi, 2001: 20: 65-77.

[19] Martino Enrico, Lupi, pecore e logica, in: Filosofia e logica, M. Carrara, P. Giaretta (a cura di), 103-33, Rubettino, Catanzaro, 2004.

[20] Panza Marco, Sereni Andrea, Il problema di Platone. Un'introduzione storica al la filosofia del la matematica, Carocci, Roma, 2010.

[21] Pettigrew Richard, Platonism and Aristotelianism in Mathematics, Pbilosophia Mathematica, 2008: 16:310-332.

[22] Schirn Matthias, Fregean abstraction, referential indeterminacy and the logical foundations of arithmetic, Erkenntnis, 2003: 59: 203-232.

[23] Shapiro Stewart, Philosophy of Mathematics: Structure and Ontology, Oxford University Press, Oxford, New York, 1997.

[24] Shapiro Stewart, Identity, Indiscernibility, and Ante Rem Structuralism: The Tale of $i$ and -i, Philosophia Mathematica, 2008: 16: 285-309.

[25] Shapiro Stewart, An " $i$ ” for an $i$ : Singular Terms, Uniqueness, and Reference, Review of Symbolic Logic, 2012: 5: 380-415.

[26] Stirton William, Caesar invictus, Philosophia Mathematica, 2003: 11: 285-304.

[27] Stirton William, Caesar and Circularity, in: Abstractionism: Essays in Philosophy of Mathematics P.A. Ebert, M. Rossberg (a cura di), 39-49, Oxford University Press, New York, 2017.

[28] Woods Jack, Logical Indefinites, Logique et Analyse, 2014: 227: 277-307.

[29] Wright Crispin, Frege's Conception of Numbers as Objects, Aberdeen University Press, Aberdeen, 1983.

[30] Zalta Edward, Frege's Theorem and Foundations for Arithmetic, Stanford Encyclopedia of Philosophy, URL $=<$ https://plato.stanford.edu/archives/sum 2017/entries/frege-theorem/>, 1998, edizione rivista 2017. 